\title{
Feasibility of the utilization of BNCT in the fast neutron therapy beam at Fermilab
}

\author{
Katja Langen ${ }^{1,2}$, Arlene J. Lennox ${ }^{2,3}$, Thomas K. Kroc ${ }^{2}$ and Paul M. DeLuca, \\ $\mathrm{Jr}^{1}$ \\ ${ }^{1}$ University of Wisconsin, Department of Medical Physics, Madison, Wisconsin; ${ }^{2}$ Neutron Therapy Facil- \\ ity at Fermilab, Batavia, Illinois; and ${ }^{3}$ Saint Joseph Hospital, Elgin, Illinois, USA
}

Keywords: BNCT, dose boost, neutron therapy.

\section{Introduction}

The Neutron Therapy Facility at Fermilab has treated cancer patients since 1976. Since then more than 2,300 patients have been treated and a wealth of clinical information accumulated. The therapeutic neutron beam at Fermilab is produced by bombarding a beryllium target with $66 \mathrm{MeV}$ protons. The resulting continuous neutron spectrum ranges from thermal to $66 \mathrm{MeV}$ in neutron energy. It is clear that this spectrum is not well suited for the treatment of tumors with boron neutron capture therapy (BNCT) only. However, since this spectrum contains thermal and epithermal components we are investigating whether BNCT can be used in this beam to boost the tumor dose. There are clinical scenarios in which a selective tumor dose boost of $10-15 \%$ could be clinically significant. For these cases the principal treatment would still be fast neutron therapy but a tumor boost could be used either to deliver a higher dose to the tumor tissue or to reduce the dose to the normal healthy tissue while maintaining the absorbed dose level in the tumor tissue.

\section{Clinical rationale}

The clinical rationale for this study is based on the poor prognosis of certain brain tumor patients. Following diagnosis the expected survival time for patients diagnosed with glioblastoma multiforme is around 8 months and can be extended by another 4 months with radiation therapy. There is no difference in survival between fast neutron and photon therapy patients, but the cause of death does depend on the treatment modality. A regrowth of the tumor is the primary cause of death in photon patients whereas with fast neutron therapy one achieves tumor control but patients sustain a high degree of normal tissue damage that causes death [1]. These clinical results indicate a small therapeutic ratio of glioma

Address for correspondence: Katja Langen, University of Wisconsin-Madison, Department of Medical Physics, 1530 MSC, 1300 University Avenue, Madison, WI 53703, USA. 
to normal brain tissue. In order to increase the survival the therapeutic ratio must be increased. BNCT has the potential to achieve just that in fast neutron beams.

A selective dose enhancement in the tumor tissue will allow a corresponding reduction in the overall absorbed dose level, therefore reducing the normal tissue complications.

\section{Materials and Methods}

We are investigating modification of the existing neutron energy spectrum by filtration in order to increase the low energy component of the beam thus enhancing the boron neutron capture dose boost. Monte Carlo calculations are used to simulate the effects of various filter materials. Microdosimetric techniques are used to measure the boron dose enhancement in a given beam. The ultimate choice of the filter assembly will depend on the irradiation technique used, e.g., will BNCT be administered simultaneously with fast neutron therapy or in a separate session? Demands on the filter assembly change with irradiation technique. The choice of technique will on the other hand be dictated by the effects that can be achieved with different filter materials.

Monte Carlo calculations were performed using MCNP [2]. For some selected materials, data files were available that extended to $100 \mathrm{MeV}$ [3]. These files were used for the following calculations. The neutron energy spectrum for the Fermilab facility was calculated by Ross et al. [4]. We used this neutron fluence spectrum as a source for our calculations. Neutrons were emitted into a cone to simulate a collimated neutron field. At the isocenter distance we modeled a cylindrical water phantom that is surrounded by $5 \mathrm{~mm}$ of bone surface, to simulate a head. Slabs of filter materials were placed adjoining to the head phantom. Figure 1 shows a schematic of the beam and phantom model. Monte Carlo scor-

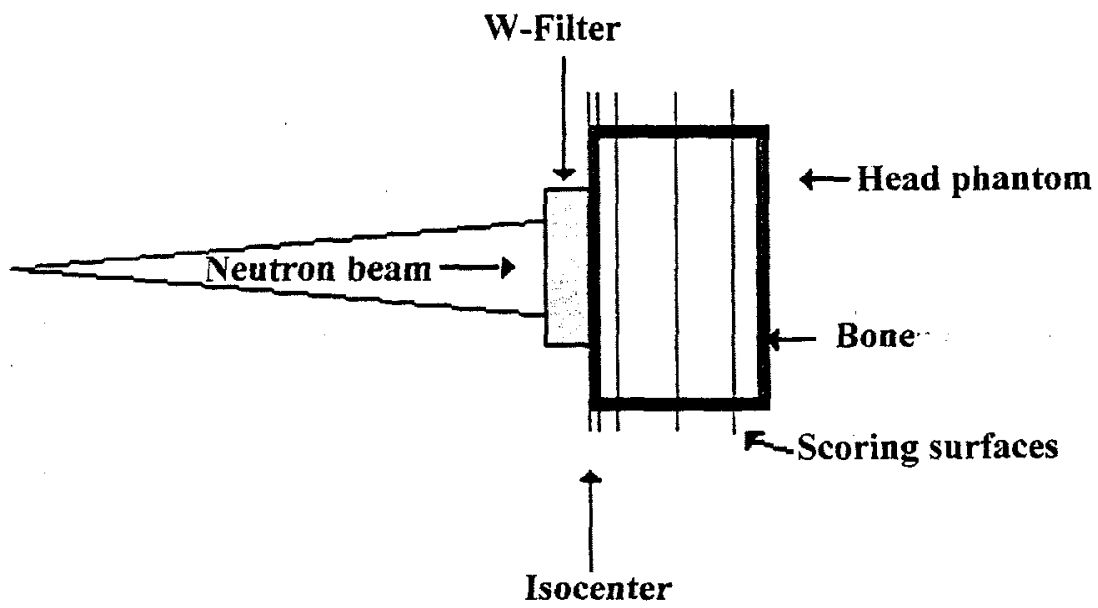

Fig. 1. Schematic of the geometry used for the Monte Carlo calculations. 
ing surfaces were set at the bone and brain surface as well as at various depths in the head phantom. Several filter materials were investigated; tungsten having the most favorable effects on the neutron energy spectrum. Figure 2 shows Monte Carlo results obtained at $50 \mathrm{~mm}$ depth in the head phantom for several tungsten filter thicknesses. Spectra are normalized to the highest energy bin. These results indicate that the thermal and epithermal neutron rates increase with the tungsten filter thickness, however, the dose rate reduction due to the tungsten filters will limit the acceptable tungsten filter thickness. A dose rate reduction by a factor of 10 is expected for a $100-\mathrm{mm}$ tungsten filter.

Microdosimetry can be employed to directly measure the dose boost in the neutron beam using the following technique: in order to simulate a boronated tumor, a proportional counter was constructed with an A-150 tissue-equivalent plastic wall that is loaded with $200 \mathrm{ppm}{ }^{10} \mathrm{~B}$. This counter is simultaneously irradiated with a regular A-150 tissue-equivalent plastic counter that is identical to the boronated counter in every aspect except the boron additive to the wall. A difference in counter responses is then due to the boron additive in the one counter wall.

\section{Preliminary results}

Initial measurements and calculations were completed. Monte Carlo calculations indicate a potential for tungsten filtration of the beam. Therefore, a $90-\mathrm{mm}$ thick tungsten filter was used to modify the neutron beam. Figure 3 shows the microdosimetric spectra measured in the filtered beam at $50-\mathrm{mm}$ water depth. An equal area under a microdosimetric spectra is equal to an equal absorbed dose

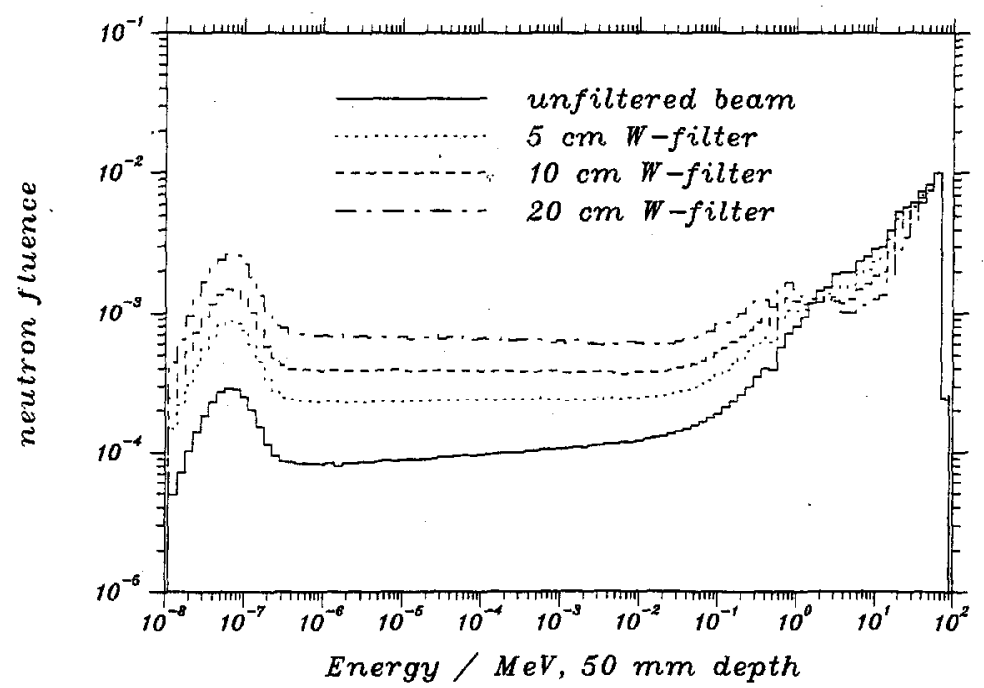

Fig. 2. Monte Carlo results calculated for $50 \mathrm{~mm}$ depth in the head phantom. 


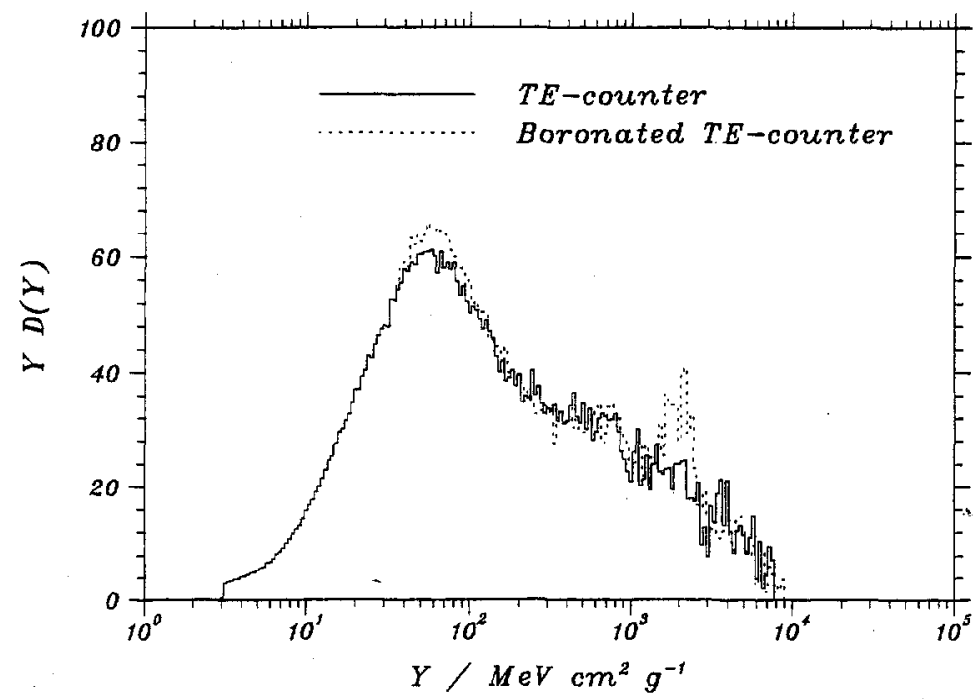

Fig. 3. Microdosimetric spectra measured in the neutron therapy beam at Fermilab. The beam was filtered by $90 \mathrm{~mm}$ of tungsten.

in the wall material. Analysis of the data indicates a $5-7 \%$ boron dose enhancement as measured with the $200 \mathrm{ppm}$ loaded counter.

The measurement confirms the usefulness of tungsten as a beam filtration material. However, future measurements will focus on further increasing the boron dose enhancement. Besides beam filtration we will investigate the effects of beam reflectors as well as the use of different materials for beam collimation.

\section{References}

1. Saroja KR, Mansell J, Hendrickson FR, Cohen L, Lennox AJ. Failure of accelerated neutron therapy to control high grade astrocytomas. Int J Radiat Oncol Biol Phys 1989;17:1295-1297.

2. Briesmeister FJ (ed) MCNP 4 A general Monte Carlo code for neutron transport and photon transport. Los Alamos National Laboratory 1993; LA-012625-M.

3. Young PG, Arthur ED, Bozoain M, England TR, Hall GM, LaBauve RJ, Little RC, MacFarlane RE, Madland DG, Perry RT, Wilson WB. Transport data libraries for incident proton and neutron energies to $100 \mathrm{MeV}$. Los Alamos National Laboratory 1990; LA-11753-MS.

4. Ross MA, DeLuca PM, Jr., Jones DTL, Lennox AJ, Maughan RL. Calculated fluence spectra at neutron therapy facilities. Radiat Prot Dosim (In press). 